\title{
A combined HM-PCR/SNuPE method for high sensitive detection of rare DNA methylation
}

\author{
Sascha Tierling ${ }^{* 1}$, Matthias Schuster ${ }^{2}$, Reimo Tetzner ${ }^{3}$ and Jörn Walter ${ }^{1}$
}

\begin{abstract}
Background: DNA methylation changes are widely used as early molecular markers in cancer detection. Sensitive detection and classification of rare methylation changes in DNA extracted from circulating body fluids or complex tissue samples is crucial for the understanding of tumor etiology, clinical diagnosis and treatment. In this paper, we describe a combined method to monitor the presence of methylated tumor DNA in an excess of unmethylated background DNA of non-tumorous cells. The method combines heavy methyl-PCR, which favors preferential amplification of methylated marker sequence from bisulfite-treated DNA with a methylation-specific single nucleotide primer extension monitored by ion-pair, reversed-phase, high-performance liquid chromatography separation.

Results: This combined method allows detection of $14 \mathrm{pg}$ (that is, four to five genomic copies) of methylated chromosomal DNA in a 2000-fold excess (that is, $50 \mathrm{ng}$ ) of unmethylated chromosomal background, with an analytical sensitivity of $>90 \%$. We outline a detailed protocol for the combined assay on two examples of known cancer markers (SEPT9 and TMEFF2) and discuss general aspects of assay design and data interpretation. Finally, we provide an application example for rapid testing on tumor methylation in plasma DNA derived from a small cohort of patients with colorectal cancer.

Conclusion: The method allows unambiguous detection of rare DNA methylation, for example in body fluid or DNA isolates from cells or tissues, with very high sensitivity and accuracy. The application combines standard technologies and can easily be adapted to any target region of interest. It does not require costly reagents and can be used for routine screening of many samples.
\end{abstract}

\section{Background}

Changes in DNA methylation such as hypermethylation of tumor suppressor genes are regarded as early molecular events during cancer development. Such epigenetic changes are widely used as molecular markers in tumor cell diagnostics [1-3]. Selective and robust PCR-based screening methods for very early detection of abnormal tumor-specific methylation become increasingly important. In particular, methods to screen for the low abundance of aberrantly methylated tumor DNA present in peripheral blood samples or other body fluids are regarded as very promising, non-invasive, early cancer detection tests [4].

Although many potentially diagnostic DNA methylation markers for such sensitive tests have been identified

* Correspondence: s.tierling@mx.uni-saarland.de

1 Universität des Saarlandes, FR. 8.3 Biowissenschaften, Genetik/Epigenetik, 66041 Saarbrücken, Germany

Full list of author information is available at the end of the article for several solid tumor types (for example, colon, breast or prostate cancer) only a few have passed validation tests in larger clinical studies on blood plasma samples [5-9]. One reason not to gain faster progress in 'biomarker' validation is the lack of robust and versatile methods for comprehensive and robust clinical testing. A combination of real-time PCR using either methylation-specific primers (MS-PCR)[10] or blockers (heavy methyl-PCR; HMPCR) [11] with methylation-specific fluorescence probes (for example, MethyLight) [12] have successfully been used to detect low copy numbers of aberrantly methylated tumor DNA in blood plasma samples [12,13]. In this paper, we present a novel assay combining sensitive HMPCR with methylation-restricted single nucleotide primer extension (MR-SNuPE) followed by ion-pair, reversed-phase, high-performance liquid chromatography IIP/RP-HPLC) detection. The combined assay has the highest analytical sensitivity, allows an excellent product and quality monitoring, and is as sensitive as real- 
time PCR-based assays. Technically the assay is easy to perform, including semi-automatable processing steps (see workflow outlined in Figure 1). It requires a HPLC system but otherwise operates at relatively low cost, as it does not rely on the use of complex chemistry. We demonstrate the sensitivity and application of the method to

Extraction

(5 $\mathrm{mL}$ of blood plasma, Lysis, DNA capturing on magnetic particles)

\section{Bisulfite conversion}

(Sodium bisulfite based treatment in thermo cycler over night)
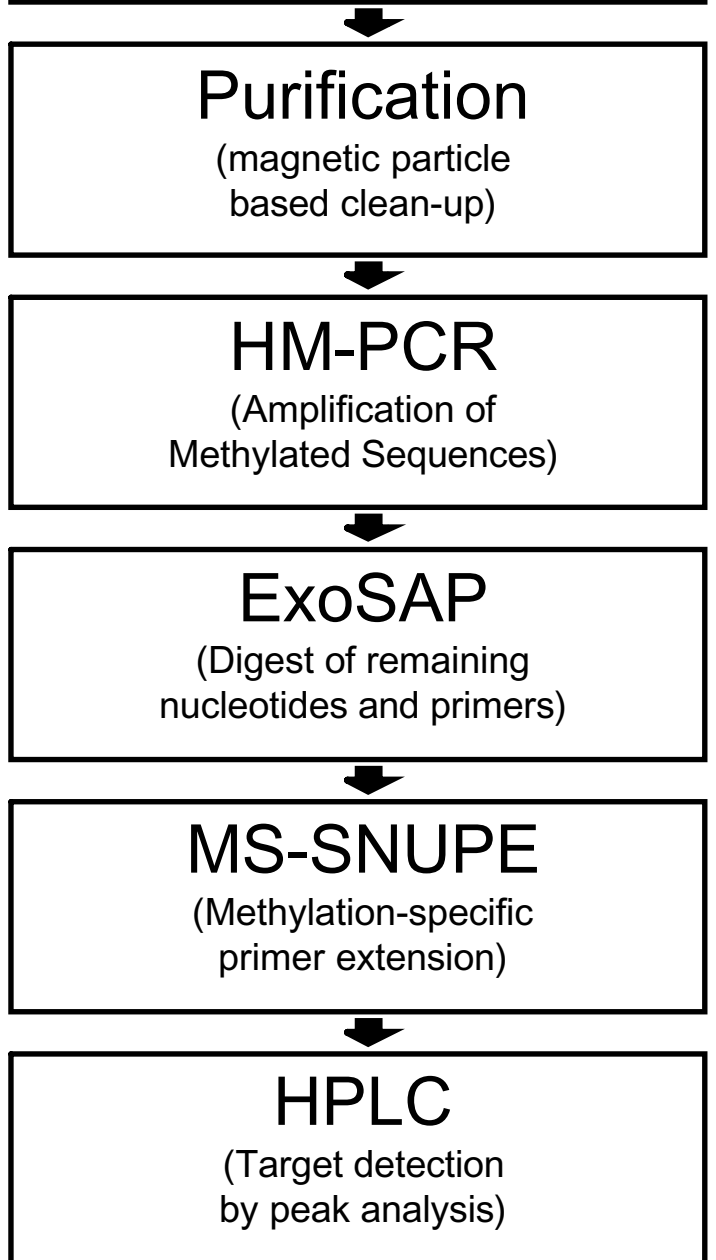

Figure 1 Workflow of the combined heavy methyl (HM)-PCR/ methylation-restricted single nucleotide primer extension (MRSNuPE) assay. routine diagnostics in a pilot study on two pre-validated colon cancer methylation markers, transmembrane epidermal growth factor (TMEFF2/TPEF) and septin 9 (SEPT9) [14-18].

\section{Results}

We first determined the limits of SNuPE detection by IP/ RP-HPLC on PCR products obtained by conventional PCR of bisulfite-treated template DNA. We mixed decreasing amounts of fully methylated template (1:1 to $1: 1,000)$ in an excess of fully unmethylated bisulfitetreated DNAs. These mixed template DNAs were treated with bisulfite and then used to generate SEPT9- and TMEFF2-specific PCR products. Amplicons were subsequently treated with an exonuclease I/shrimp alkaline phosphatase (SAP) mix to remove PCR primers and dephosphorylate dNTPs, followed by heat inactivation and direct use of CpG-specific primer extension (see workflow Figure 1). Primers were designed for both strands of the PCR products, and extension reactions were performed with regular unlabelled ddNTPs. For extension primers we used either the CpG-free (unbiased recognition of bisulfite DNA; MS-SNuPE)[19] primers 'oligo 27' for SEPT9 and 'oligo 30' for TMEFF2, or the (methylation-restricted; MR-SNuPE) CpG dinucleotidecontaining primers 'oligo 45 ' for SEPT9 and 'oligo 62' for TMEFF2 (top strand) and 'oligo 28' for SEPT9 and 'oligo 44' for TMEFF2 (bottom strand) (Figure 2A; see Additional file 1 , supplemental figure $1 \mathrm{~A}$ ). SNuPE reaction products were individually separated on a column (DNASep $^{\text {tw }}$ Cartridge; Transgenomic, Omaha, NE, USA) using a DNA fragment analyzer (WAVE ${ }^{\mathrm{ma}}$ DNA Fragment Analysis System; Transgenomic). Figure 2B shows the MS-SNuPE and MR-SNuPE electropherograms obtained for the SEPT9-specific extensions (primers 27, 28 and 45). Product peaks (ultraviolet spectra at $260 \mathrm{~nm}$ ) representing methylated and unmethylated templates (designated $\mathrm{M}$ and $\mathrm{UM}$ in the figures) were identified on fully methylated and unmethylated amplicons, respectively (Figure 2B ( $a$ and $b)$ ). We recommend performing such control reactions to standardize experimental series because absolute retention times can change depending on the age of the HPLC column. Moreover, a no-template control (NTC) was carried along with each MS-SNuPE and MR-SNuPE reaction to reveal the retention time of the unextended primer and unspecific primer products (for example, see oligo 45 in Figure 2B). The incremental dilutions of methylated templates in an unmethylated background revealed small but reproducibly detectable methylation-specific primer extensions even in mixtures containing only one methylated copy in 100 unmethylated copies (that is, a detection limit of about 1\%). Although the detection of methylation was robust for all primers, the presence of unmethylated products obvi- 
A Oligo 27

gattcgttg ttattagttattatgtcggatttcgcggttaacgcgtagttggatgggattattt ctaagcaacaaataatcaataatacagcctaaagcgccaattgcgcatcaacctaccctaataaa

\section{Oligo 45}

gattcgttgtttattagttattatgtcggatttcgcggttaacgkgtagttggatgggattattt ctaagcaacaaataatcaataatacagcctaaagcgccaattgcgcatcaacctaccctaataaa

Oligo 28

gattcgttgtttattagttattatgtcggatttcgcggttaacgcgtagttggatgggattattt ctaagcaacaaataatcaataatacagectaaagcgccaattgcgcatcaacctaccctaataaa

B a fully methylated

b fully unmethylated template

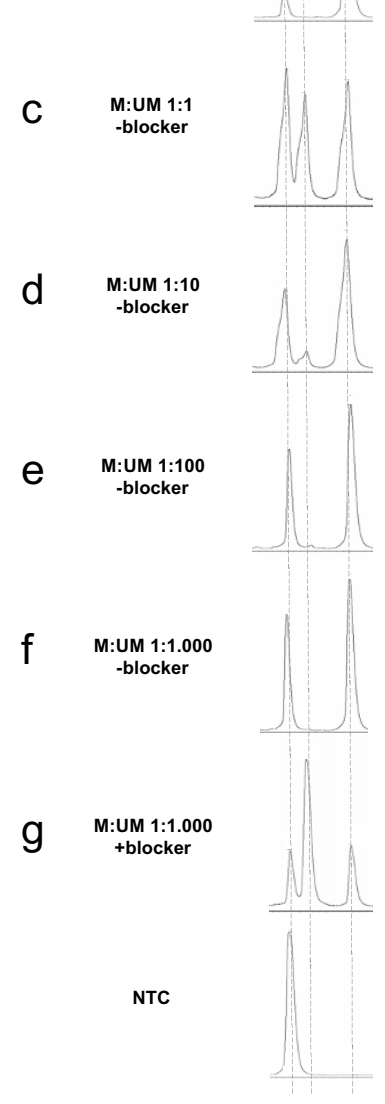

UP M UM
Oligo 27

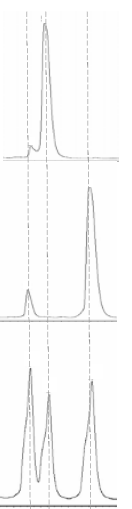

Oligo 45

Oligo 28
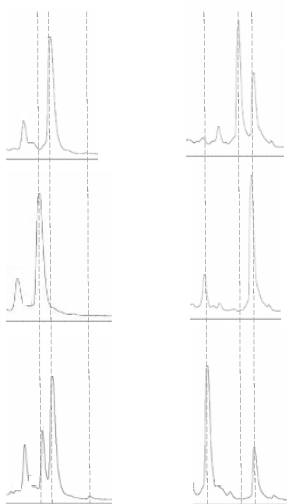

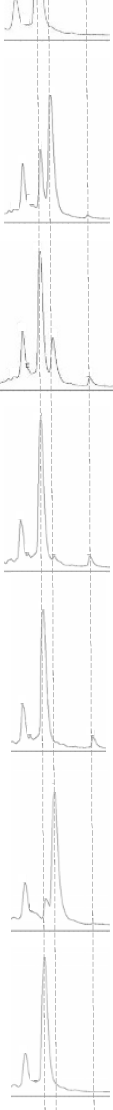

UP M UM

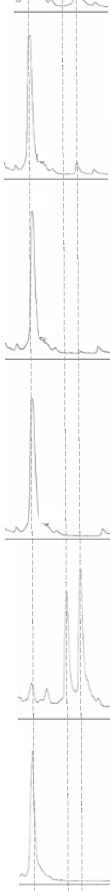

UP M UM

Figure 2 MR-SNuPE assay design for SEPT9 and general performance. (A) SEPT9 amplicon sequence with indicated primer (boxes) and blocker (line above) positions. (B) Electropherograms of separated single nucleotide primer extension (SNuPE) products from PCR products obtained without blocker on (a) completely methylated and (b) unmethylated DNA templates or (c-f) mixed DNA templates (methylated:unmethylated DNA ratios/ dilution series). (g) Effect when performing a heavy methyl (HM)-PCR; that is, when the blocker is included, on a dilution shown in (f). Vertical dashed lines indicate the positions of unextended primer, methylated and unmethylated signals, respectively, NTC = no template control, that is, SNuPE reaction without PCR template. 
ously influences the sensitivity and specificity of the reaction. For example, unmethylated extension products were obtained with oligo 28 and oligo 45, which should only bind to and extend methylated templates (Figure 2B). Analogous results were obtained for TMEFF2-specific reactions (see Additional file 1, supplemental figure 1B). To suppress unspecific extension and enhance the detection sensitivity, a PCR blocker (for position within the PCR products see Figure 2A, Additional file 1, supplemental figure $1 \mathrm{~A}$ ) which specifically binds and suppresses the amplification of unmethylated templates, was included in the PCR reactions. The presence of this blocker significantly enhances the enrichment of methylated products. Clear methylation-specific extension products (designated as M; Figure 2B (g); see Additional file 1 , supplemental figure $1 \mathrm{~A}(\mathrm{~g})$ ) were visible down to a 1:1,000 dilution. Nevertheless, the blocker does not completely suppress the amplification of unmethylated templates, which could be visualized as primer extension products when using the oligos $27,30,62,44$ and 28 , which produce unmethylated extension products (UM in the figure). Note that the signals representing unmethylated products for oligo 28 were at least partially caused by its complementarity to the blocker that is present in the reaction, hence, the presence of the blocker should be taken into account when performing primer design.

In summary, the analysis performed on two amplicons revealed that MR-SNuPE reactions performed on templates generated by conventional unbiased PCR already reach high levels of detection, that is, at least one methylated copy could easily and unambiguously be detected in a background of 1,000 unmethylated copies. However, in MR-SNuPE reactions, detection of co-methylated templates is favored because oligos are used that include CpGs (oligos 28 and 45; oligos 44 and 62). Extension primers without CpGs (oligos 27 and 30) bind independently of the methylation state (MS-SNuPE reaction) and might not be as sensitive but allow simultaneous detection of co-amplified non-methylated templates and, hence, serve as a good HM-PCR (or MS-PCR) quality control (see below).

To enhance the sensitivity we then combined SNuPE reactions on PCR products produced by HM-PCR. To define the limit of detection in such double selective reactions for very low DNA concentrations such as in blood plasma DNA samples, we again performed a proof of principle experiment. We spiked defined amounts of methylated templates $(6.25,12.5,25,50$ and $100 \mathrm{pg})$ into unmethylated background (50 ng) and performed primer extension reactions on 10 to $24 \mathrm{HM}-\mathrm{PCR}$ reactions for each 'dilution'. For SEPT9 we observed a 100\% detection down to $25 \mathrm{pg}$ of spiked methylated template (corresponding to 1 methylated copy in 500 to 2,000 unmethylated copies) (see Additional file 1, supplemental Figure
2A, supplemental Figure 2B and supplemental table 1). A detection rate of $83.3 \%$ was obtained with $12.5 \mathrm{pg}$ ( 1 in $4,000)$ and $58.3 \%$ with $6.25 \mathrm{pg}(1$ in 8,000$)$ methylated template. Hence, with a $90 \%$ probability (Probit analysis), the method allows detection of $13.6 \mathrm{pg}$ (CI 9.6 to $193 \mathrm{pg}$ ) of methylated copies in an excess of unmethylated background for SEPT9 and $21.9 \mathrm{pg}$ (CI 13.0 to $36.9 \mathrm{pg}$ ) for TMEFF2 (Additional file 1, supplemental Figure 2C, supplemental Figure 2D). Hence, the combined HM-PCR/ $\mathrm{MR}-\mathrm{SNuPE}$ assay reaches sensitivities close to the theoretical optimum.

In a double-blind study, we then used the combined HM-PCR/MR-SNuPE assay to detect methylation at SEPT9 in free-floating tumor DNA isolated from blood plasma of colon cancer probands. One-fifth of bisulfitetreated DNA samples isolated from 4-5 $\mathrm{ml}$ of plasma taken from 20 patients with colorectal cancer (CRC) and 20 healthy donors was used for HM-PCR and subsequent MS-SNuPE and MR-SNuPE. In all 20 healthy donor samples, only unmethylated products were seen, whereas in the CRC samples, methylated products were found in 10 of the 20 cases by HM-PCR/primer extension assays using oligo 27 and oligo 45 (Figure 3). In a real-time coupled HM-PCR assay, 11 of the 20 CRC samples were found to be methylation-positive (data not shown) [11]. Both methods together detected 13 of 20 cases as methylation-positive, but were discordant for five samples: two cases were clearly detected by HM-PCR/MR-SNuPE and not by real-time HM-PCR alone, and the other three cases were only positive by real-time HM-PCR. When analyzing the threshold cycles $(\mathrm{CT})$ of the corresponding real-time PCRs, we observed that methylated DNA detection was in all cases close to the single copy level in the respective samples (data not shown). The discordant detection of five cases is therefore most likely a result of stochastic PCR variability on extremely low concentrations of methylated DNA in the respective plasma samples. In fact, both the real-time HM-PCR and the HM$\mathrm{PCR} / \mathrm{SNuPE}$ assays were performed on different aliquots of the same bisulfite-treated DNA. Thus, we recommend performing assays in replicates to decrease the number of false negatives resulting from limited amounts of methylated copies. Overall, our pilot experiment shows that there is no significant difference in the performance and sensitivity of both methods (a McNemar test for both methods on the 20 CRC samples showed a similar performance with $P=1.0$ ).

\section{Discussion}

By combining two highly selective DNA methylation enrichment techniques (HM-PCR and MR-SNuPE), we generated a novel assay with extremely sensitive detection rates. With this combination, we were able to detect 14 pg methylated template DNA ( four to five haploid 


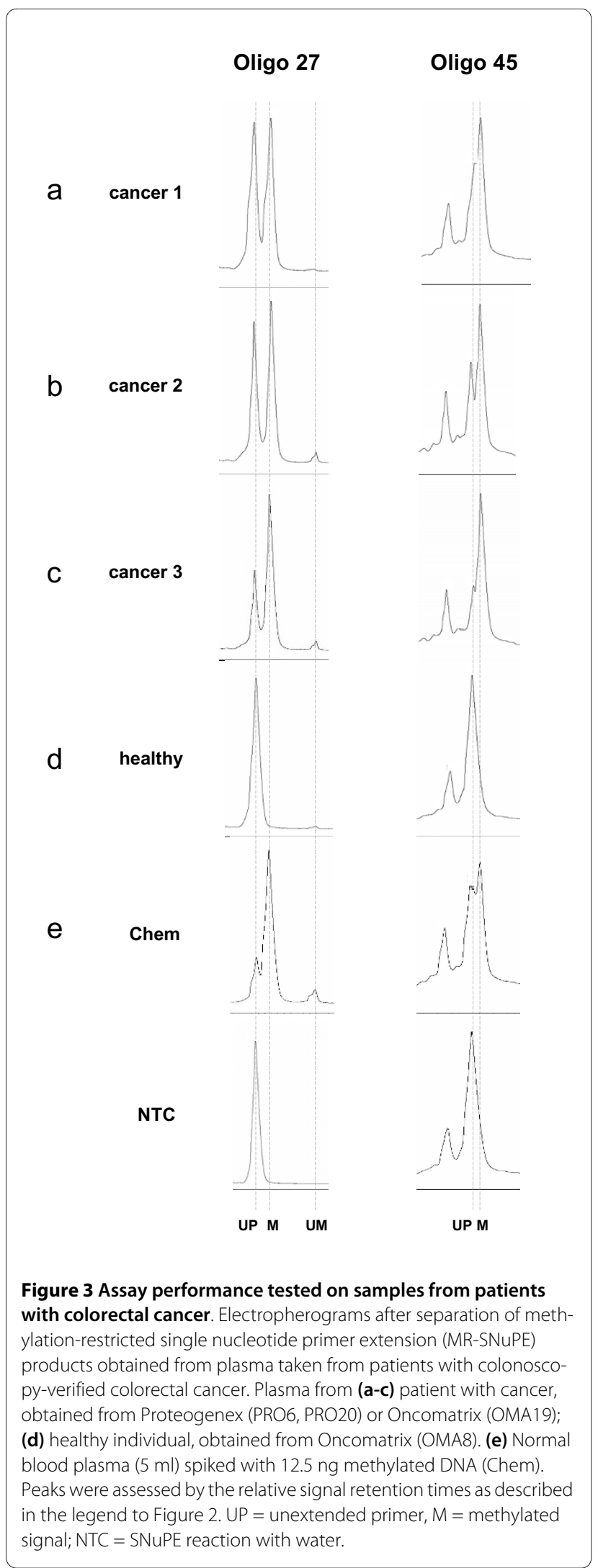

copies) in $50 \mathrm{ng}$ of unmethylated DNA. The method reached a detection level close to the theoretical limit. We then performed a small pilot study on plasma DNA samples from 20 patients with CRC and 20 healthy individuals to demonstrate that the method can in principle be used for routine analysis in biomedical research.

All of the main assays such MS-PCR, MethyLight or HM-PCR used for the detection of rare methylation in DNA samples are based on selective bisulfite-PCR amplification, but differ in the manner of data readout and the level of sensitivity [20-23]. In comparison to the realtime-based methods, MethylLight and other HM-PCRbased assays, the combined HM-PCR/MR-SNuPE is a simple end-point analysis that is comparable with MSPCR but is more sensitive and selective. It does not require sophisticated chemistry but still achieves an excellent detection level. Moreover, by using combinations of MS-SNuPE and MR-SNuPE primers, it is possible to assess several CpG positions independently and also to quality control the selective methylation-specific PCR performance (see below). Very recently, another alternative assay reaching a similar sensitivity was reported. That method uses heat-stable restriction enzymes during PCR [24]. By contrast, our method is not limited to the presence of distinct restriction sites in the amplicon of interest and, hence, is more flexible in its application. A further advantage is that it does not require extensive purification steps or complex and costly nucleotide chemistry for the analysis.

In our experience, the combination of SNuPE assays with HM-PCR assays (and presumably MS-PCR also) requires very little HPLC optimization. However, great care should be taken with primer design for both MS$\mathrm{SNuPE}$ and MR-SNuPE reactions. We recommend using 12-16 nucleotides long HPLC-purified oligos. To achieve the highest selectivity and sensitivity in MR-SNuPE reactions, the oligos should encompass at least three $\mathrm{CpG}$ dinucleotides. In addition, any complementarity with the blocker used in HM-PCR should be avoided. For optimum HPLC separation, primers should be generated from the A-rich strand to generate either ddCTP or ddTTP extensions. Although the number of methylationspecific CpG positions in the extension oligos (such as oligo 45 that includes four CpGs) enhances the specificity for methylated products only, the detection sensitivity is equally good with oligos that additionally detect coamplified non-methylated templates in the HM-PCR reaction (see oligo 27 in Figure 2B (g), for example). We recommend including at least one unbiased (MS-SNuPE) extension primer (oligos 27 and 30 in our example) in the assay. This allows simultaneous detection of unmethylated and methylated products, serves as a direct quality control for the HM-PCR (MS-PCR) amplification, and 
monitors the accuracy of the SNuPE extension. Besides the high sensitivity and specificity, the combination of HM-PCR and SNuPE detection has several practical advantages for routine sensitive diagnostics. First, the inclusion of a blocker for unmethylated DNA into the HM-PCR reaction significantly reduces the amount of unmethylated byproducts and enhances the sensitivity and specificity of the SNuPE detection. A similar principle is used in MS-PCR assays when primers are placed across several methylated $\mathrm{CpG}$ positions [10]. In contrast to MS-PCR, the use of unbiased primers and the presence of selective blockers in our assay reduce but do not eliminate the amplification of unmethylated DNA. Moreover, the assay does not require complete co-methylation of all CpG positions. Secondly, our approach represents an all-or-nothing detection, that is, methylated DNA templates (above the detection threshold) always provide strong signals/peaks so that misinterpretations (false positives) can almost invariably be excluded.

Because the detection limit may easily be reached in plasma DNA samples (as seen in our small study) and because individual DNA samples might stochastically vary in the amount of tumor DNA present, the problem of false negatives has to be considered. We therefore suggest performing reactions in triplicate and, if possible, on independent DNA isolates and on several genes/amplicons. We are convinced that this robust combined HMPCR/MR-SNuPE assay will be a useful diagnostic tool for the fast and cost-efficient detection of rare DNA methylation and will be applicable to larger cohort studies.

\section{Conclusions}

The described HM-PCR/MR-SNuPE assay is a robust and versatile endpoint analysis method for high sensitive detection of rare DNA methylation in complex DNA samples. The assay is cost-efficient and allows semi-automated processing of samples, making it applicable for routine testing in DNA samples isolated from biopsy tissues or body fluids.

\section{Methods}

\section{DNA and sample material}

Universal in vitro methylated human DNA was obtained from Millipore. Unmethylated DNA was prepared by molecular displacement amplification (MDA)[25] of 10 ng peripheral blood leukocyte DNA (Promega, Mannheim, Germany) using a commercial kit (Repli-G Kit; Qiagen, Hilden, Germany). Blood plasma from 20 patients with CRC (stages I, II, III) and 20 colonoscopyverified normal controls was obtained from Oncomatrix and ProteoGenex, where samples were collected after informed consent and in compliance with local guidelines.

\section{Sample preparation}

DNA was isolated from $5 \mathrm{ml}$ of blood plasma and bisulfite converted [26] using previously established procedures (for detailed information see Additional file 1).

\section{Real-time PCR}

HM-PCR was performed in 96-well plates on a thermal cycler (LightCycler 480; Roche, Mannheim, Germany). For both SEPT9 and TMEFF2, each $25 \mu \mathrm{l}$ reaction contained $300 \mathrm{nM}$ each of both forward and reverse primers (Table 1), $1 \mu \mathrm{M}$ blocker and $100 \mathrm{nM}$ of probe in $1 \times$ buffer (QuantiTect mPCR Kit NoROX; Qiagen, Hilden, Germany). After 30 minutes at $95^{\circ} \mathrm{C}, 50$ cycles of PCR were performed: 30 seconds at $56^{\circ} \mathrm{C}$ and 10 seconds at $95^{\circ} \mathrm{C}$ for SEPT9; 30 seconds at $56^{\circ} \mathrm{C}, 10$ seconds at $72^{\circ} \mathrm{C}, 10 \mathrm{sec}-$ onds at $95^{\circ} \mathrm{C}$ for $T M E F F 2$. The mixture was then cooled to $40^{\circ} \mathrm{C}$

\section{HM-PCR, primer extension and HPLC separation}

For methylation analysis by SNuPE, HM-PCR was performed as described above, omitting the probe. An aliquot $(5 \mu \mathrm{l})$ of the HM-PCR product were treated with $1 \mu \mathrm{l}$ of Exo-SAP (1:10 mixture of exonuclease I and SAP (USB, Staufen, Germany) for $30 \mathrm{~min}$ at $37^{\circ} \mathrm{C}$. To inactivate the Exo-SAP enzymes, the reaction was incubated for 15 minutes at $80^{\circ} \mathrm{C}$. Primer extension was carried out as described previously[27].

For SEPT9, to the PCR product/Exo-SAP mix, $2 \mu \mathrm{l}$ of $10 \times$ buffer C (Solis BioDyne), 2.4 $\mu \mathrm{l}$ of $30 \mu \mathrm{M}$ SNuPE primer (oligos 27, 28 and 45), $1 \mu \mathrm{l}$ of $1 \mathrm{mM}$ ddCTP and ddTTP or ddGTP and ddATP, respectively, and $0.5 \mu \mathrm{l}$ of Termipol DNA polymerase ( $5 \mathrm{U} / \mu \mathrm{l}$, Solis BioDyne) were added to reach a final volume of $20 \mu \mathrm{l}$. Reactions were performed in a thermal cycler under the following conditions: $96^{\circ} \mathrm{C}$ for 2 minutes, followed by 50 cycles $96^{\circ} \mathrm{C}$ for 30 seconds, $50^{\circ} \mathrm{C}$ for 30 seconds and $60^{\circ} \mathrm{C}$ for 2 minutes. Separation of SNuPE products was conducted at $50^{\circ} \mathrm{C}$ by continuously mixing buffer $\mathrm{B}(0.1 \mathrm{M}$ triethylammonium acetate (TEAA), $25 \%$ acetonitrile) to buffer A $(0.1 \mathrm{M}$ TEAA) over 10 minutes, resulting in a buffer B concentration of $28 \%$ to $35 \%$ for oligo $27,20 \%$ to $27 \%$ for oligo 28 , and $21 \%$ to $27 \%$ for oligo 45 .

For TMEFF2, To the PCR product. Exo-SAP mix, $2 \mu \mathrm{l}$ of $10 \times$ buffer $C$ (Solis BioDyne), $2.4 \mu \mathrm{l}$ of $30 \mu \mathrm{M}$ SNuPE primer (oligos 30, 44 and 62), $1 \mu \mathrm{l}$ of $1 \mathrm{mM}$ ddCTP and ddTTP or ddGTP and ddATP, respectively, and $0.5 \mu \mathrm{l}$ of Termipol ( $5 \mathrm{U} / \mu \mathrm{l}$; Solis BioDyne) were added to reach a final volume of $20 \mu \mathrm{l}$. Reactions were performed in a thermal cycler under the following conditions: $96^{\circ} \mathrm{C}$ for 2 minutes followed by 50 cycles of $96^{\circ} \mathrm{C}$ for 30 seconds, $60^{\circ} \mathrm{C}$ for 30 seconds and $60^{\circ} \mathrm{C}$ for 2 minutes. Separation of $\mathrm{SNuPE}$ products was conducted at $50^{\circ} \mathrm{C}$ by continuously mixing buffer $B$ (0.1 M TEAA, 25\% acetonitril) to buffer A (0.1 M TEAA) over 10 minutes, resulting in a buffer $B$ 
Table 1: Oligos used in the assays

\begin{tabular}{|c|c|c|}
\hline Gene name & Oligo name & Sequence 5'T3' \\
\hline \multirow[t]{7}{*}{ SEPT9 } & Forward & GATT-X-GTTGTTTATTAGTTATTATGT \\
\hline & Reverse & AAATAATCCCATCCAACTA \\
\hline & Blocker & $\begin{array}{l}\text { GTTATTATGTTGGATITTGTGGTTAATGTGT } \\
\text { AG-C3 }\end{array}$ \\
\hline & Probe & FAM-TTAACCGCGAAATCCGAC-BHQ1 \\
\hline & Oligo 27 & ttattagttattatgt \\
\hline & Oligo 28 & cggatttcgcggttaacg \\
\hline & Oligo 45 & cgcgttaaccgcgaaatcc \\
\hline \multirow[t]{7}{*}{ TMEFF2 } & Forward & GGTTATTGTTTGGGTTAATAAATG \\
\hline & Reverse & AAAAAAAAAAAACTCCTCTACATAC \\
\hline & Blocker & $\begin{array}{l}\text { ACATACACCACAAATAAATTACCAAAAAC } \\
\text { ATCAACAA-C } 3\end{array}$ \\
\hline & Probe & FAM-TTCGGACGTCGTTGTTCGG-BHQ1 \\
\hline & Oligo 30 & gttaataaatggagtt \\
\hline & Oligo 44 & gaacaacgacgtcc \\
\hline & Oligo 62 & cgtcgttgttcggt \\
\hline
\end{tabular}

$\mathrm{X}=$ tetrahydrofuran-type abasic site

concentration of $21 \%$ to $29 \%$ for oligo $30,16 \%$ to $26 \%$ for oligo 44 , and $18 \%$ to $28 \%$ for oligo 62 .

\section{Cloning and bisulfite sequencing of methylated and unmethylated SEPT9 amplicons}

For preparation of methylated and unmethylated SEPT9 amplicons, $5 \mathrm{ng}$ of bisulfite-treated methylated and unmethylated DNA, respectively, were amplified by HM-PCR without using a probe. PCR products were cloned into pGemT vector (Promega, Mannheim, Germany) according to the manufacturer's protocol, and transformed into Escherichia coli TOP10 cells. Positive clones were checked by colony PCR and subsequently sequenced. Complete unmethylated and methylated clones were used for HPLC separation.

\section{Limit of detection}

In total, $50 \mathrm{ng}$ of bisulfite-treated unmethylated DNA was spiked with subnanogram amounts (100, 50, 25, 12.5, $6.25,0 \mathrm{pg}$ ) of bisulfite-treated methylated DNA and analyzed in replicate by both methods in parallel. The limit of detection was defined as the minimum amount of bisulfite-treated methylated DNA that could be distinguished from unmethylated background with $90 \%$ confidence.

\section{Additional material}

Additional file 1 Supplementary material. 


\section{Competing interests}

JW is a cofounder of Epigenomics AG, Berlin and works as a scientific advisor for the company. RT is employee and shareholder of the Epigenomics AG. SEPT9 is a diagnostic biomarker commercialized by Epigenomics AG. ST and MS declare no conflict of interest.

\section{Authors' contributions}

ST, MS, RT and JW made substantial contributions to conception and design of the study. ST designed and performed the SNuPE experiments. MS and RT designed and performed HM-PCR experiments. All authors read and approved the final manuscript.

\section{Acknowledgements}

We are grateful for the technical support provided by Transgenomic for the WAVE IP/RP-HPLC analyses. This work was supported by the BMBF program BioChancePlus \#0313166.

\section{Author Details}

IUniversität des Saarlandes, FR. 8.3 Biowissenschaften, Genetik/Epigenetik, 66041 Saarbrücken, Germany, 2Dx assays, Blk 35 Marsiling Ind Estate Road 3 Woodlands Central Industrial Estate, 739257, Singapore and ${ }^{3}$ Epigenomics AG, Kleine Präsidentenstr. 1, 10178 Berlin, Germany

Received: 20 April 2010 Accepted: 2 June 2010

Published: 2 June 2010

\section{References}

1. Ozanne SE, Constancia M: Mechanisms of disease: the developmental origins of disease and the role of the epigenotype. Nat Clin Pract Endocrinol Metab 2007, 3:539-546.

2. Gronbaek K, Hother C, Jones PA: Epigenetic changes in cancer. APMIS 2007, 115:1039-1059.

3. Esteller M: Epigenetic gene silencing in cancer: the DNA hypermethylome. Hum Mol Genet 2007, 16:R50-59.

4. Chatterjee SK, Zetter BR: Cancer biomarkers: knowing the present and predicting the future. Future Oncol 2005, 1:37-50.

5. Herman JG: Hypermethylation pathways to colorectal cancer. Implications for prevention and detection. Gastroenterol Clin North Am 2002, 31:945-958

6. Gonzalgo ML, Liang G, Spruck CH, Zingg JM, Rideout WM, Jones PA: Identification and characterization of differentially methylated regions of genomic DNA by methylation-sensitive arbitrarily primed PCR. Cancer Res 1997, 57:594-599.

7. Toyota M, Ho C, Ahuja N, Jair KW, Li Q, Ohe-Toyota M, Baylin SB, Issa JP: Identification of differentially methylated sequences in colorectal cancer by methylated CpG island amplification. Cancer Res 1999, 59:2307-2312.

8. Yan PS, Efferth T, Chen HL, Lin J, Rödel F, Fuzesi L, Huang TH: Use of CpG island microarrays to identify colorectal tumors with a high degree of concurrent methylation. Methods 2002, 27:162-169.

9. Model F, Osborn N, Ahlquist D, Gruetzmann R, Molnar B, Sipos F, Galamb O, Pilarsky C, Saeger HD, Tulassay Z, Hale K, Mooney S, Lograsso J, Adorjan P, Lesche R, Dessauer A, Kleiber J, Porstmann B, Sledziewski A, Lofton-Day C: Identification and validation of colorectal neoplasia-specific methylation markers for accurate classification of disease. Mol Cancer Res 2007, 5:153-163.

10. Herman JG, Graff JR, Myohänen S, Nelkin BD, Baylin SB: Methylationspecific PCR: a novel PCR assay for methylation status of $\mathrm{CpG}$ islands. Proc Natl Acad Sci USA 1996, 93:9821-9826.

11. Cottrell SE, Distler J, Goodman NS, Mooney SH, Kluth A, Olek A, Schwope I, Tetzner R, Ziebarth $\mathrm{H}$, Berlin K: A real-time PCR assay for DNAmethylation using methylation-specific blockers. Nucleic Acids Res 2004, 32:e10.

12. Eads CA, Danenberg KD, Kawakami K, Saltz LB, Blake C, Shibata D, Danenberg PV, Laird PW: MethyLight: a high-throughput assay to measure DNA methylation. Nucleic Acids Res 2000, 28:e32.

13. Cottrell SE, Laird PW: Sensitive detection of DNA methylation. Ann NY Acad Sci 2003, 983:120-130.

14. Liang G, Robertson KD, Talmadge C, Sumegi J, Jones PA: The gene for a novel transmembrane protein containing epidermal growth factor and follistatin domains is frequently hypermethylated in human tumor cells. Cancer Res 2000, 60:4907-4912.

15. Sabbioni S, Miotto E, Veronese A, Satin E, Gramantieri L, Bolondi L, Calin GA, Gafà R, Lanza G, Carli G, Terrazzi E, Feo C, Liboni A, Rullini S, Negrini M: Multigene methylation analysis of gastrointestinal tumors: TPEF emerges as a frequent tumor-specific aberrantly methylated marker that can be detected in peripheral blood. Mol Diagn 2003, 7:201-207.

16. Grützmann R, Molnar B, Pilarsky C, Habermann JK, Schlag PM, Saeger HD Miehlke S, Stolz T, Model F, Roblick UJ, Bruch HP, Koch R, Liebenberg V, Devos T, Song X, Day RH, Sledziewski AZ, Lofton-Day C: Sensitive detection of colorectal cancer in peripheral blood by septin 9 DNA methylation assay. PLoS One 2008, 3:e3759.

17. Lofton-Day C, Model F, Devos T, Tetzner R, Distler J, Schuster M, Song X, Lesche R, Liebenberg V, Ebert M, Molnar B, Grützmann R, Pilarsky C, Sledziewski A: DNA methylation biomarkers for blood-based colorectal cancer screening. Clin Chem 2007, 54:414-423.

18. DeVos T, Tetzner R, Model F, Weiss G, Schuster M, Distler J, Vaughn-Steiger K, Grützmann R, Pilarsky C, Habermann JK, Day R, Sledziewski A, LoftonDay C: Circulating methylated septin 9 in plasma is a biomarker for colorectal cancer. Clin Chem 2009, 55:1337-1346.

19. Gonzalgo ML, Jones PA: Rapid quantitation of methylation differences at specific sites using methylation-sensitive single nucleotide primer extension (Ms-SNuPE). Nucleic Acids Res 1997, 25:2529-2531.

20. Xiong Z, Laird PW: COBRA - a sensitive and quantitative DNA methylation assay. Nucleic Acids Res 1997, 25:2532-2534.

21. Weber M, Davies JJ, Wittig D, Oakeley EJ, Haase M, Lam WL, Schübeler D: Chromosome-wide and promoter-specific analyses identify sites of differential DNA methylation in normal and transformed human cells. Nat Genet 2005, 37:853-862.

22. Frommer M, McDonald LE, Millar DS, Collis CM, Watt F, Grigg GW, Molloy $\mathrm{PL}$, Paul CL: A genomic sequencing protocol that yields a positive display of 5-methylcytosine residues in individual DNA strands. Proc Natl Acad Sci USA 1992, 89:1827-1831.

23. Wolf $S F$, Migeon BR: Studies of $X$ chromosome DNA methylation in normal human cells. Nature 1982, 295:667-671.

24. Kneip C, Schmidt B, Fleischhacker M, Seegebarth A, Lewin J, Flemming N, Seemann S, Schlegel T, Witt C, Liebenberg V, Dietrich D: A novel method for sensitive and specific detection of DNA methylation biomarkers based on DNA restriction during PCR cycling. Biotechniques 2009, 47:737-744

25. Dean FB, Hosono S, Fang L, Wu X, Faruqi AF, Bray-Ward P, Sun Z, Zong Q, Du Y, Du J, Driscoll M, Song W, Kingsmore SF, Egholm M, Lasken RS: Comprehensive human genome amplification using multiple displacement amplification. Proc Natl Acad Sci USA 2002, 99:5261-5266.

26. Tetzner R, Dietrich D, Distler J: Control of carry-over contamination of PCR-based DNA methylation quantification using bisulfite treated DNA. Nucleic Acids Res 2007, 35:e4.

27. El-Maarri O: SIRPH analysis: SNuPE with IP-RP-HPLC for quantitative measurements of DNA methylation at specific $\mathrm{CpG}$ sites. Methods $\mathrm{Mol}$ Biol 2004, 287:195-205.

doi: 10.1186/1756-8935-3-12

Cite this article as: Tierling et al., A combined HM-PCR/SNuPE method for high sensitive detection of rare DNA methylation Epigenetics \& Chromatin 2010, 3:12 